19. Lombardi, V., Piazzesi, G., Ferenczi, M. A., Thirlwell, H. \& Irving, M. Elastic distortion of myosin heads and repriming of the working stroke in muscle. Nature 374, 553-555 (1995).

20. Piazzesi, G. et al. Changes in the $\mathrm{x}$-ray diffraction pattern from single, intact muscle fibers produced by rapid shortening and stretch. Biophys. J. 68, 92s-98s (1995).

21. Bershitsky, S. Y. et al. Mechanical and structural properties underlying contraction of skeletal muscle fibers after partial 1-ethyl-[3-(3-dimethylamino)propyl]carbodiimide cross-linking. Biophys. J. 71, 1462-1474 (1996).

22. Vainstain, B. K. Diffraction of X-rays by Chain Molecules (Izdatelstvo Akademii Nauk SSSR, Moscow, 1963)

23. Cooke, R., Crowder, M. S., Wendt, C. H., Barnett, V. A. \& Thomas, D. D. in Contractile Mechanisms in Muscle 413-423 (Plenum, New York, London, 1984).

24. Harford, J. J. \& Squire, J. M. Evidence for structurally different attached states of myosin cross-bridges on actin during contraction of fish muscle. Biophys. J. 63, 387-396 (1992).

25. Brenner, B. \& Yu, L. C. Structural changes in the actomyosin cross-bridges associated with force generation. Proc. Natl Acad. Sci. USA 90, 5252-5256 (1993).

26. Irving, M. et al. Tilting of the light-chain region of myosin during step length changes and active force generation in skeletal muscle. Nature 375, 688-691 (1995).

27. Martin-Fernandez, M. A. et al. Time-resolved X-ray diffraction studies of myosin head movements in live frog sartorius muscle during isometric and isotonic contractions. J. Musc. Res. Cell Motility 15, 319-348 (1994)

28. Towns-Andrews, E. et al. Time-resolved $\mathrm{x}$-ray diffraction station: $\mathrm{x}$-ray optics, detectors and data acquisition. Rev. Scient. Instr. 60, 2346-2349 (1989).

29. Huxley, H. E. \& Brown, W. The low-angle X-ray diagram of vertebrate striated muscle and its behaviour during contraction and rigor. J. Mol. Biol. 30, 383-434 (1967).

Acknowledgements. We thank M. Irving for comments on the early version of the manuscript, D. R Trentham for support, and E. Towns-Andrews and the non-crystalline-diffraction team at CLRC Daresbury Laboratory for hard- and sofware support. The work was supported by the MRC and grants from the EU, INTAS, ISF, HHMI, RFBR and by CCP13 of EPSRC and BBSRC

Correspondence and requests for materials should be addressed to M.A.F. (e-mail: m-ferenc@nimr.mrc. ac.uk)

\section{Inhibition of death receptor signals by cellular FLIP}

\section{Martin Irmler ${ }^{\star} \uparrow$, Margot Thome ${ }^{\star} \dagger$, Michael Hahne*, Pascal Schneider ${ }^{\star}$, Kay Hofmann $\neq$, Véronique Steiner ${ }^{\star}$, Jean-Luc Bodmer*, Michael Schröter*, Kim Burns*, Chantal Mattmann ${ }^{\star}$, Donata Rimoldi $\S$, Lars E. French\| \& Jürg Tschopp*}

* Institute of Biochemistry, $\$$ Ludwig Institute of Cancer Research, Lausanne branch, University of Lausanne, and $\ddagger$ the Swiss Institute for Experimental Cancer Research (ISREC), BIL Biomedical Research Center, Chemin des Boveresses 155, CH-1066 Epalinges, Switzerland I Department of Dermatology, University of Geneva, Medical School, CH-1211 Geneva 4, Switzerland

$\dagger$ These authors contributed equally to this work.

The widely expressed protein Fas is a member of the tumour necrosis factor receptor family which can trigger apoptosis ${ }^{1}$. However, Fas surface expression does not necessarily render cells susceptible to Fas ligand-induced death signals ${ }^{1,2}$, indicating that inhibitors of the apoptosis-signalling pathway must exist. Here we report the characterization of an inhibitor of apoptosis, designated FLIP (for FLICE-inhibitory protein), which is predominantly expressed in muscle and lymphoid tissues. The short

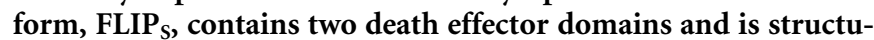
rally related to the viral FLIP inhibitors of apoptosis ${ }^{3}$, whereas the long form, FLIP $_{\mathrm{L}}$, contains in addition a caspase-like domain in which the active-centre cysteine residue is substituted by a tyrosine residue. FLIP $_{S}$ and FLIP $_{L}$ interact with the adaptor protein $\mathrm{FADD}^{4,5}$ and the protease $\mathrm{FLICE}^{6,7}$, and potently inhibit apoptosis induced by all known human death receptors ${ }^{1}$ FLIP $_{\mathrm{L}}$ is expressed during the early stage of T-cell activation, but disappears when $T$ cells become susceptible to Fas ligand-mediated apoptosis. High levels of FLIP $_{L}$ protein are also detectable in melanoma cell lines and malignant melanoma tumours. Thus FLIP may be implicated in tissue homeostasis as an important regulator of apoptosis.

We have recently described a family of six viral inhibitors ( $\mathrm{v}$ FLIPs) that are present in several $\gamma$-herpesviruses and molluscipoxvirus, and which contain two death effector domains (DEDs) ${ }^{3}$.
These inhibitors block the early signalling events of the death receptors Fas, TRAMP (wsl/DR-3/Apo-3), TRAIL-R (DR-4) and tumour necrosis factor receptor 1 (TNF-R1) (refs 1, 3, 8). v-FLIPs bind to the DED of FADD (MORT-1) and interfere with the FADDFLICE (Caspase 8/MACH/Mch-5) interaction, thereby inhibiting the recruitment and activation of FLICE by $\mathrm{Fas}^{3}$. Some v-FLIPs may also exert their inhibitory effect by binding directly to the DEDs of FLICE $^{8}$. Because many viral genes are adopted host genes, we considered the possibility that cellular homologues of the v-FLIPs may exist. We screened public databases with a generalized profile ${ }^{9}$ constructed from the six known members of the v-FLIP family and identified a partial human sequence with highly significant homology $\left(P<10^{-10}\right)$ to $\mathrm{v}$-FLIPs (Fig. 1a). When the corresponding expressed sequence tag (EST) clone was used to screen a cDNA library from activated human peripheral blood leukocytes, we isolated several cDNA clones encoding a protein with an overall structural organization similar to the v-FLIPs, which we designated FLIP $_{S}$. This protein consists of two DEDs, followed by a carboxyterminal extension of approximately 50 amino acids. An alternatively spliced cDNA coding for a longer form of FLIP, designated FLIP $_{\mathrm{L}}$, was also isolated. Within this protein the two DEDs are followed at the $\mathrm{C}$ terminus by a caspase-like domain, giving rise to a molecule structurally similar to FLICE (Fig. 1a). The gene for FLIP co-localizes with the FLICE homologue Mch4 (caspase 10$)^{10}$ to the chromosome 2q33 (ref. 11), suggesting that the genes coding for Mch4, FLIP and probably FLICE arose by gene duplication. However, the amino-acid position corresponding to the active-site cysteine of FLICE is substituted by a Tyr residue in FLIP $_{\mathrm{L}}$. The active-site cysteine was absent in all human $\mathrm{FLIP}_{\mathrm{L}}$-encoding $\mathrm{cDNA}$ clones analysed (four clones), as well as in mouse FLIP $_{\mathrm{L}}$, which had been isolated by screening a murine heart muscle cDNA library (Fig. 1a). Moreover, of the two other residues involved in catalysis ${ }^{12,13}$ and conserved in members of the caspase family, only the Gly residue is conserved (Fig. 1a); the His residue is substituted by an Arg (human FLIP $_{\mathrm{L}}$ ) or a Leu residue (mouse FLIP $_{\mathrm{L}}$ ). FLIP $\mathrm{FL}_{\mathrm{L}}$ was therefore expected to be proteolytically inactive, similar to the FLICE-1 (ref. 7) and FLICE-2 (ref. 14) mutants in which the catalytic cysteine residue had been replaced. Indeed, in contrast to FLICE, the caspase region of FLIP $_{\mathrm{L}}$ was unable to cleave two caspase-specific peptide substrates $^{7}$ (Fig. 1b). However, we cannot completely exclude the possibility that the caspase-like region of FLIP $_{\mathrm{L}}$ can act on different peptides.

Northern blots of human tissues revealed that four main FLIP RNA species exist which are predominantly expressed in heart, skeletal muscle and peripheral blood leukocytes (PBLs) (Fig. 1c). The shortest transcript (1.1-1.3 kilobases) probably corresponds to FLIP $_{S}$, as it was not detected with an RNA probe uniquely spanning the caspase region of $\operatorname{FLIP}_{\mathrm{L}}$. Three longer, less abundantly expressed transcripts reacted with both the caspase and the DED probes, and presumably corresponded to FLIP $_{\mathrm{L}}$.

Triggering of Fas results in the incorporation of v-FLIPs into the death-inducing signalling complex (DISC) ${ }^{3,15}$. To assess whether cellular FLIP was functionally similar to v-FLIP, we analysed its interaction with FADD, which connects Fas with FLICE during death signal transduction ${ }^{6,7}$. Indeed, both human FLIP $_{\mathrm{L}}$ (relative molecular mass $\left(M_{\mathrm{r}}\right)$ of $\left.\sim 55 \mathrm{~K}\right)$ and $\operatorname{FLIP}_{\mathrm{S}}\left(M_{\mathrm{r}} \sim 28 \mathrm{~K}\right)$ associated strongly with FADD (Fig. 2a) when coexpressed in 293T cells. The FLIP $_{L}-$ FADD interaction was mediated by the DEDs of FLIP $_{L}$, as the caspase-like region of $\operatorname{FLIP}_{L}\left(\right.$ FLIP $\left._{c}\right)$ displayed no affinity for FADD. In cells transfected with expression vectors for FLIP $_{S}$, FLIP $_{L}$, FADD and the cytoplasmic portion of Fas, a stable Fas-FADDFLIP $_{L}$ (or FLIP ) complex was formed, indicating that both forms of FLIP can incorporate into the DISC of Fas.

Current evidence suggests that precursor caspases are activated and autoprocessed as a dimeric complex, ultimately resulting in a stable $(\mathrm{p} 10 / \mathrm{p} 20)_{2}$ complex $^{12,13,16}$. Correspondingly FLICE overexpression in $293 \mathrm{~T}$ cells resulted in the formation of dimers or 
higher oligomers of FLICE (the experimental conditions do not allow the distinction between these two possibilities) (Fig. 2b). We therefore anticipated that the FLICE-like FLIP $_{\mathrm{L}}$ would also form homodimers. However, FLIP $_{\mathrm{L}}$ dimers (or oligomers) were not detectable (Fig. 2c). In contrast, FLIP $_{\mathrm{L}}$ interacted with FLICE (Fig. 2d), indicating that heterodimers can be formed between functional (FLICE) and non-functional $\left(\mathrm{FLIP}_{\mathrm{L}}\right)$ caspases. The presence of FLICE in immunoprecipitates of FLIP $_{S}$ and of FLIP (the caspase-like region of $\mathrm{FLIP}_{\mathrm{L}}$ ) revealed that both the N-terminal DEDs, as well as the C-terminal caspase-like region of $\mathrm{FLIP}_{\mathrm{L}}$, contribute to the interaction with FLICE (Fig. 2d).

Overexpression of FLIP $\mathrm{L}_{\mathrm{L}}$ in $293 \mathrm{~T}$ cells in the absence of z-VADfmk (a caspase inhibitor which had been included in the experiments described above) resulted in the appearance of two molecular species. One of these corresponds to the expected molecular size of full-length $\operatorname{FLIP}_{\mathrm{L}}\left(M_{\mathrm{r}} \sim 55 \mathrm{~K}\right)$, and the second is a C-terminally truncated form of $\operatorname{FLIP}_{\mathrm{L}}\left(M_{\mathrm{r}} \sim 43 \mathrm{~K} ; \mathrm{FLIP}_{43}\right)$, which arises from cleavage after Asp 376 of FLIP $_{\mathrm{L}}$ (data not shown) corresponding to the p17-p12 boundary in other related caspase members (Figs. 1a, 2e). Initial processing of FLICE during Fas-mediated activation occurs at the corresponding site ${ }^{17}$. Precursor FLICE is a natural substrate of active FLICE ${ }^{6,17}$, and it is therefore likely that the FLICElike FLIP $_{\mathrm{L}}$ is processed by endogenous FLICEs. Processing of fulllength FLIP $_{\mathrm{L}}$ into the $43 \mathrm{~K}$ form was also observed when Raji B cells, stably transfected with $\mathrm{FLIP}_{\mathrm{L}}$ (see below), were treated with soluble Fas ligand (sFasL) (Fig. 2e). The cleavage of FLIP $_{\mathrm{L}}$ is reminiscent of the cleavage of the viral apoptosis inhibitor p35 (refs 18, 19), which is an excellent substrate for several caspases, yet its cleavage results in a tighter binding to the caspases. Similarly, FLIP $_{\mathrm{L}}$ cleavage through receptor-activated FLICE may result in a strong FLIP $_{L}-$ FLICE interaction, thereby blocking further processing and activation of FLICE. This model, together with the FLIP interaction data (Fig. 2), predicts that FLIP is capable of arresting death receptor signals. This was validated experimentally in $293 \mathrm{~T}$ cells, where the extensive cellular death induced by the overexpression of Fas was efficiently reduced by coexpression of $\operatorname{FLIP}_{\mathrm{L}}$ (Fig. 3a) and $\mathrm{FLIP}_{\mathrm{S}}$ (data not shown). Moreover, coexpression of FLIP $_{S}$ or FLIP $_{L}$, but not of FLIP (the caspase-like domain of FLIP $_{\mathrm{L}}$; see Fig. 1a), protected 293T cells from apoptosis induced by TRAMP (DR3/wsl/Apo-3) ${ }^{20,23}$ (Fig. 3b), indicating that the presence of the DEDs within FLIP $_{\mathrm{L}}$ is essential for

a

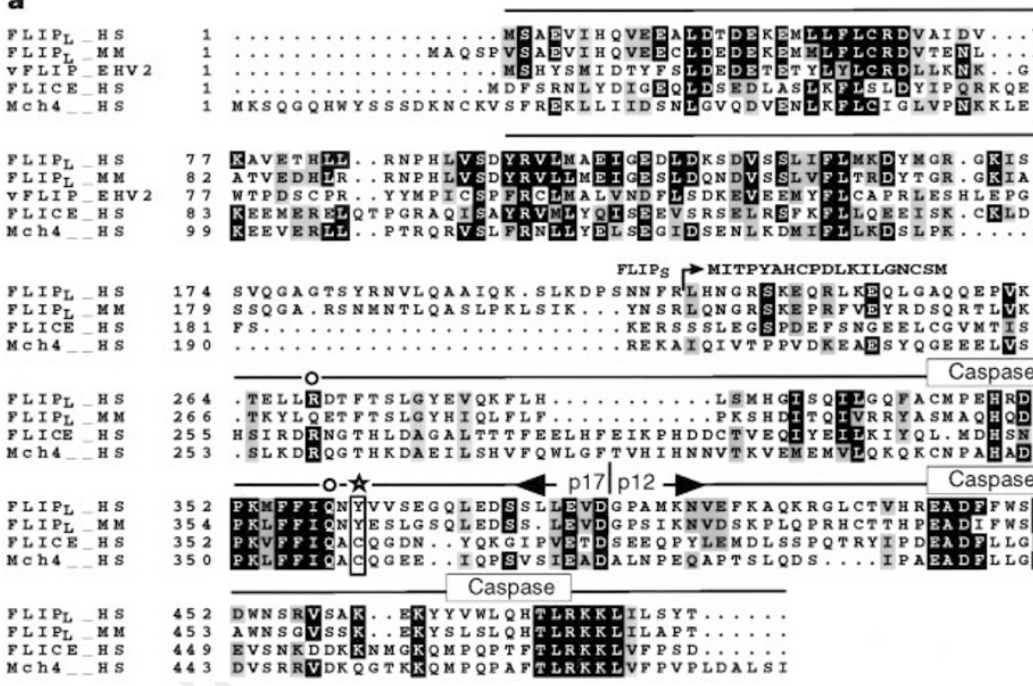

Figure 1 a, Amino-acid sequence alignment of human (HS) and mouse (MM) FLIPs, the viral FLIP (v-FLIP) from equine herpesvirus-2 (EHV2) (GenBank accession no. U20824), FLICE (ref. 6) and Mch4 (ref. 10). The long form of FLIP (FLIPL) contains two DEDs and a caspase-like domain, linked by a region of $\sim 65$ amino acids. The sequence of the alternatively spliced short form of FLIP (FLIPS) includes the two DEDs. Thus, the first 202 amino acids of the long and the short form of human FLIP are identical; only the unique C-terminal 19 amino acids of FLIPS are shown here. A typical splice consensus sequence is found at the site of divergence between FLIPL and FLIP . For each block of aligned sequences, black boxes indicate $>50 \%$ amino-acid sequence identity; while grey shading indicates $>50 \%$ sequence similarity through conservative aminoacid substitutions. The positions corresponding to the residues of FLICE and Mch4 involved in catalysis $^{12,13}$ (His, Gly and Cys) are marked by a star, and the active-centre Cys is boxed. Open circles indicate caspase residues constituting the binding pocket for the carboxylate chain of the $\mathrm{P} 1$ Asp residue ${ }^{12,13}$. The boundary of the $\mathrm{p} 17$ and $\mathrm{p} 12$ subunits of FLICE ${ }^{6,7}$ and the start of the caspase region-containing construct of FLIPL $\left(F L I P_{c}\right)$ are indicated. $\mathbf{b}$, The caspase homology region of FLIPL lacks protease activity. Cleavage kinetics of the poly(ADP ribose) polymerase (PARP)-derived fluorogenic substrate, Ac-DEVD-AMC $(50 \mu \mathrm{M})$ by the caspase homology region of FLICE (Ser 217 through the $C$ terminus; filled symbols) and of FLIP $(A$ la 233 through the $\mathrm{C}$ terminus; open symbols). Both proteins failed to cleave the fluorogenic substrate AcYVAD-AMC (ICE-cleavage site in the interleukin-1 $\beta$ precursor; data not shown). c, Tissue distribution of the FLIP transcripts. Northern blots $\left(2 \mu \mathrm{g}\right.$ poly $\left(\mathrm{A}^{+}\right) \mathrm{RNA}$ per lane) of various human tissues were probed with antisense RNA covering the N-terminal sequence of FLIP $P_{L}$ containing the two DEDs (FLIP(N), top) and the C-terminal caspase-like domain (FLIP(C), bottom), respectively. Arrowheads indicate FLIP transcripts detected only by the FLIP(N) probe (open arrows) or by both probes (FLIP(N) and FLIP(C), filled arrows). The blots were subsequently hybridized with a $\beta$-actin probe.
DED I

V P P NVR R

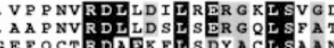

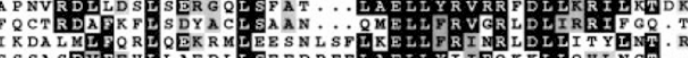
DED II

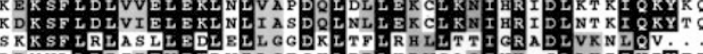

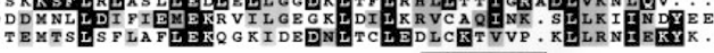
$\overrightarrow{P F L I P}_{\mathrm{C}}--$ Caspase

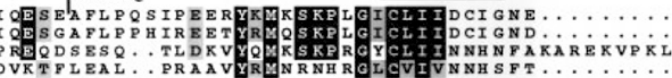

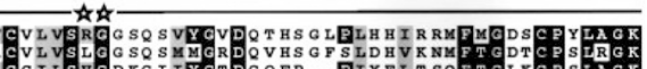

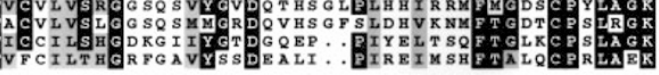
TaD 0

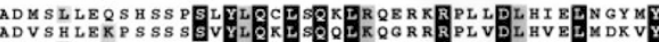

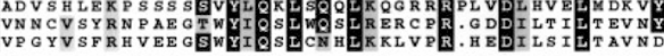

b
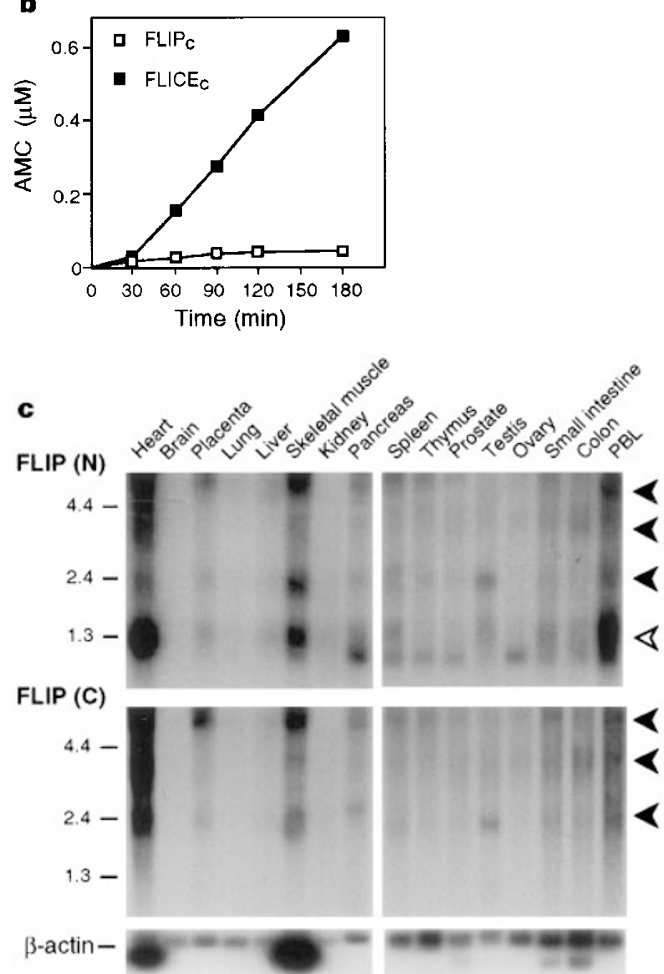
the protective function of $\mathrm{FLIP}_{\mathrm{L}}$. $\mathrm{FLIP}_{\mathrm{L}}$ and $\mathrm{FLIP}_{\mathrm{S}}$ also protected $293 \mathrm{~T}$ cells against apoptosis induced by overexpression of the cytoplasmic domain of TNF-R1 (ref. 24), albeit less efficiently (Fig. 3c and data not shown). Surprisingly, the transfection of high concentrations of FLIP $_{\mathrm{L}}$ expression vector alone (but not of FLIP $_{S}$ ) led to spontaneous cell death in 293T cells (Fig. 3d), raising the possibility that expression levels of FLIP $_{\mathrm{L}}$ may determine the life or death of a cell. We therefore stably transfected Raji B and Jurkat $\mathrm{T}$ cell lymphomas with $\mathrm{FLIP}_{\mathrm{L}}$ or $\mathrm{FLIP}_{\mathrm{S}}$ (Fig. 3e, f). In all clones obtained (and melanomas and T cells; see below), FLIP protein levels were at least 10-fold lower than the levels achieved in FLIPexpressing 293T cells undergoing apoptosis (Fig. 3e), suggesting that the extremely high concentration of FLIP observed in transfected $293 \mathrm{~T}$ cells may not be physiological. The amount of FLIP protein present in stably transfected lymphocyte clones correlated well with the relative resistance to sFasL-induced cell death (Fig. 3e, f). Western blot analyses of FLIP $\mathrm{S}_{\mathrm{S}}$ and $\mathrm{FLIP}_{\mathrm{L}}$ in cells that showed equal levels of protection against apoptosis consistently revealed that FLIP $_{\mathrm{L}}$ was a more potent inhibitor of cell death than was FLIP (Fig. 3e, f). In the Jurkat clone JFS5, for example, the relative level of FLIP $_{S}$ protein was higher than the level of FLIP $\mathrm{L}_{\mathrm{L}}$ expressed in Jurkat clone JFL2 or JFL1. However, only the FLIP $_{\mathrm{L}}$-expressing clones were protected against sFasL-induced apoptosis (the Fas surface expression was identical in all FLIP-expressing clones; data not shown). In contrast, the FLIP levels of JFS5 were sufficient to partly protect the clone against sTRAIL-induced apoptosis (Fig. 3e), indicating that, relative to Fas, the TRAIL-R signalling pathway is more effectively blocked. In view of the recent observation that TRAIL-R does not recruit $\mathrm{FADD}^{25}$, this suggests that FLIP may preferentially interact with an unknown DED-containing protein specifically recruited by the TRAIL-R or, alternatively, that a second FADD-interacting
TRAIL-R exists. FLIP expression did not modulate apoptosis triggered by various other stimuli, such as the kinase inhibitor staurosporine (Fig. 3g), or growth factor withdrawal (data not shown).

FLIP was able to protect lymphoma cell lines against death receptor-induced apoptosis, so we investigated whether FLIP was implicated in the resistance of $\mathrm{T}$ lymphocytes against FasLmediated apoptosis observed during early stages of activation ${ }^{2}$. In accordance with published data ${ }^{2}$, Fas-expressing human $\mathrm{T}$ cells were resistant to sFasL-induced apoptosis one day after mitogen stimulation despite there being abundant surface-exposed Fas levels, whereas they became sensitive after an additional 5 days in culture (Fig. 4a). This transient resistance correlated with FLIP expression levels (Fig. 4a). At day one, a 55K anti-FLIP-reactive band was detected at a position identical to that found in FLIP $_{\mathrm{L}}$-transfected, z-VAD-fmk-treated 293T cells. In FasL-responding T cells activated for 6 days, the level of expression of FLIP had decreased considerably. An anti-FLIP reactive protein migrating at $\sim 28 \mathrm{~K}$ (corresponding to $\mathrm{FLIP}_{\mathrm{S}}$ ) was not detected, despite the presence of high FLIP $_{S}$ RNA levels in PBLs (see Fig. 1c). These results suggest that FLIP $_{S}$ expression may be controlled at the post-transcriptional level. Similar results were obtained with mitogen (concanavalin A)activated mouse splenocytes (Fig. $4 \mathrm{~b}$ ). Both the $55 \mathrm{~K}$ and the processed $43 \mathrm{~K}$ forms of FLIP $_{\mathrm{L}}$ were present in these cells at day one of stimulation, whereas FLIP $_{\mathrm{L}}$ was no longer detectable in FasLsensitive lymphocytes analysed 3 days after stimulation (Fig. 4b). Thus activation of $\mathrm{T}$ cells induces a transient resistance to Fasinduced apoptotic signals that correlates with increased FLIP expression. FasL-resistant activated T cells fail to recruit and activate FLICE $^{26}$ as would be predicted if FLIPs played a major role in this protection. a

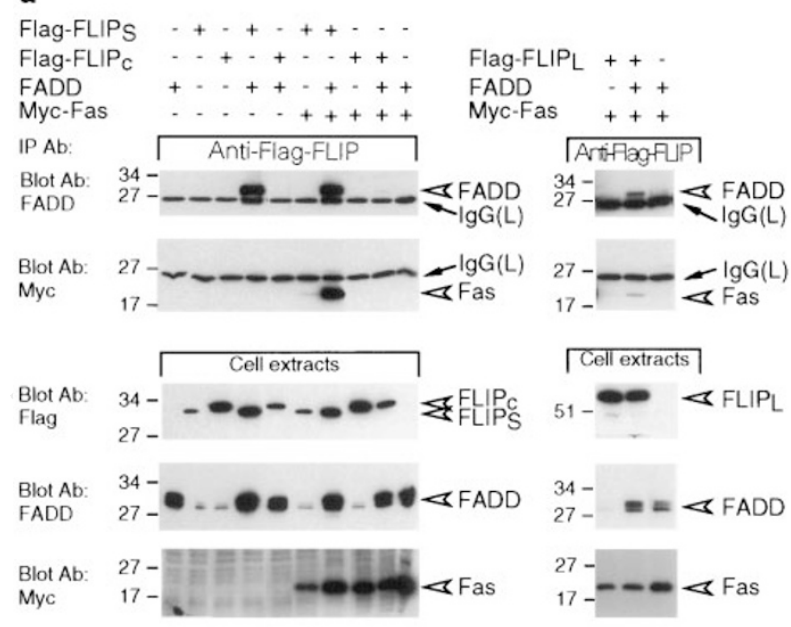

b

HA-FLICE $+\cdots++\cdots$

FLICE $-++\cdots++$

IP Ab: $H A$

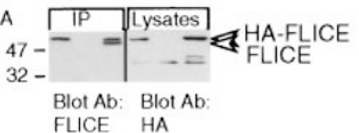

c

Flag-FLIPL $+\cdots+++$

VSV-FLIPL +++++

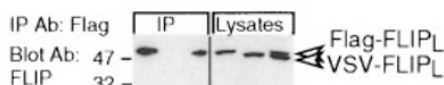

BLIP

32 d

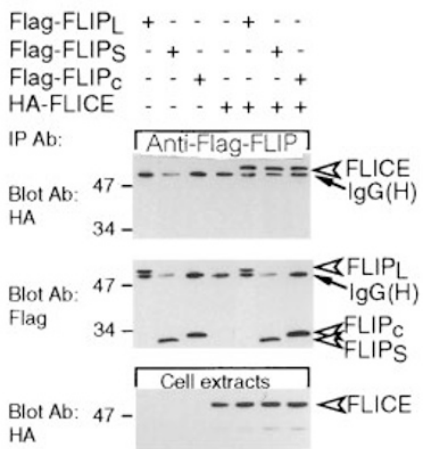

\section{e}

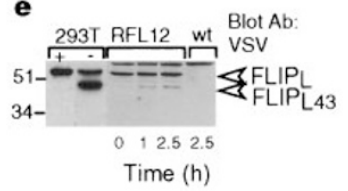

Figure 2 FLIPs interact with FADD and FLICE. a, 293T cells were transiently transfected with expression vectors encoding Flag-FLIPS, Flag-FLIP , Flag-FLIP $_{c}$ (covering the caspase-like region of $F L I P L), F A D D$ and the myc-tagged cytoplasmic domain of Fas. In these and the following experiments, appropriate quantities of vector alone were added to keep the total amount of transfected plasmid constant and $25 \mu \mathrm{M} z$-VAD-fmk was added to FLIP transfections (except in e). Cells were lysed $30 \mathrm{~h}$ after transfection, and anti-Flag immunoprecipitates (IP) or total cell extracts were analysed for the presence of FADD, Flag-FLIP or Myc-Fas by western blotting. b, Western blot analysis of anti-HA-FLICE immunoprecipitates and cell lysates from 293T cells, transfected with expression vectors encoding HA-FLICE and FLICE as indicated. c, Western blot analysis of anti-Flag immunoprecipitates and cell lysates from 293T cells transiently transfected with expression vectors encoding Flag-FLIP $L$ and VSV-FLIP. d, Anti-Flag-FLIP immunoprecipitates and cell extracts from Flag-FLIP ${ }_{L}$, Flag-FLIP,
Flag-FLIP ${ }_{c}$ and HA-FLICE transfected 293T cells were analysed by anti-HA and anti-Flag western blotting. e, FLIP $\mathrm{L}$ processing in sFasL-treated Raji cells. 293T cells were incubated in the absence $(-)$ or presence $(+)$ of the caspase inhibitor $z-V A D$-fmk. In the absence of the inhibitor, FLIPL $(55 K)$ is partly processed to a 43K fragment $\left(F L P_{43}\right)$ in $293 T$ cells. A Raji B lymphoma clone stably transfected with $N$ terminally VSV-tagged FLIP (clone RFL12; see Fig. 3f) and the parental clone (Raji wt) were treated for the indicated times with $1 \mu \mathrm{g} \mathrm{ml}^{-1}$ of recombinant sFasL. Cells were lysed and processing of VSV-FLIP $L$ was analysed by anti-VSV western blotting 1 and $2.5 \mathrm{~h}$ after the addition of SFasL. The presence of SFasL leads to partial processing of FLIP into a $43 \mathrm{~K} \mathrm{~N}$-terminal fragment. A protein migrating at $\sim 60 \mathrm{~K}$ is detected nonspecifically. The FLIPL-transfected Raji clone is resistant to apoptosis (see Fig. 3), whereas more than 90\% of parental Raji cells have an apoptotic phenotype $2.5 \mathrm{~h}$ after the addition of SFasL. 
Finally, we investigated whether FLIP is expressed in certain tumour cell lines that are resistant to death receptor-mediated apoptosis $^{27}$. None of the melanoma cell lines tested here or in a previous report ${ }^{27}$ respond to sFasL (Fig. 4c), despite the fact that some cell lines express surface Fas (Fig. 4c, d). In contrast to FasLsensitive Jurkat cells, the melanoma cell lines express detectable levels of the $55 \mathrm{~K}$ form of $\mathrm{FLIP}_{\mathrm{L}}$ protein (Fig. 4e) and mRNA (detected by reverse transcription-polymerase chain reaction (RT-PCR); data not shown). Furthermore, FLIP was expressed in metastatic cutaneous melanoma lesions from human patients (five were examined to date, and all express FLIP) (Fig. 4f; data not shown). In contrast, no FLIP was detected in melanocytes surrounding the hair follicle of the skin (Fig. 4f), indicating that FLIP upregulation probably occurs during tumorigenesis. FLIP expression may therefore contribute to the resistance to FasL of melanoma cell lines which express surface Fas.

Death receptor-induced apoptosis is an important event in tissue homeostasis as exemplified by the accumulation of $\mathrm{T}$ cells in Fasdeficient mice ${ }^{1}$. Therefore, a tight regulation of the balance between resistance and susceptibility of cells towards apoptosis is required. In $\mathrm{T}$ cells, this resistance is transient to avoid premature activationinduced cell death and to allow T cell-mediated help or cytotoxicity. In contrast, certain tumour cells seem to have lost the capacity to undergo apoptosis, either as a result of the loss of certain death receptors or by an impaired signalling pathway. Unlike the Bcl-2 family members which are potent inhibitors of apoptosis induced by growth factor withdrawal and $\gamma$-irradiation, FLIP seems to primarily block apoptosis induced by death receptors. In the examples analysed here ( $\mathrm{T}$ cells and melanomas), FLIP expression correlates with the transient or acquired resistance to apoptosis, suggesting that FLIP may contribute to the blockade of the death signalling pathways in these cells. Highest FLIP mRNA levels are detected in the heart. The administration of anti-Fas antibodies into mice causes death from liver failure, not heart failure, despite the a

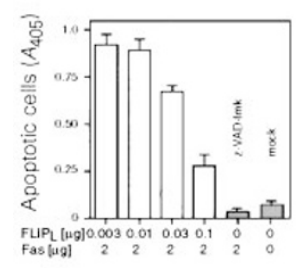

b

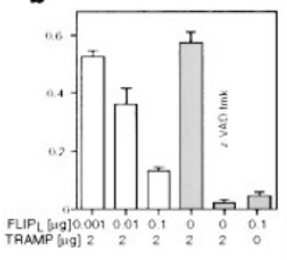

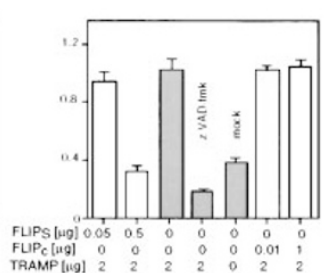

c

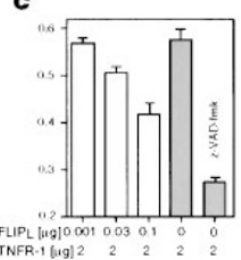

d

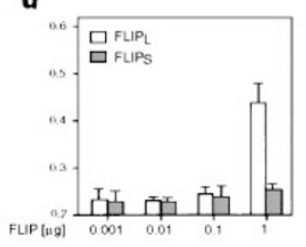

$\mathbf{e}$
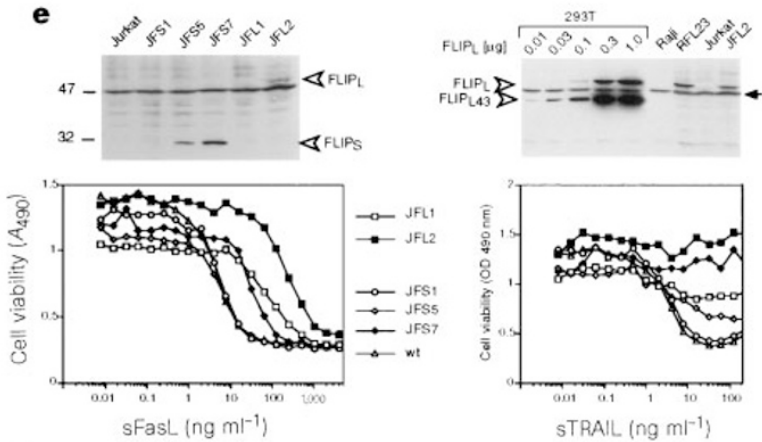

g

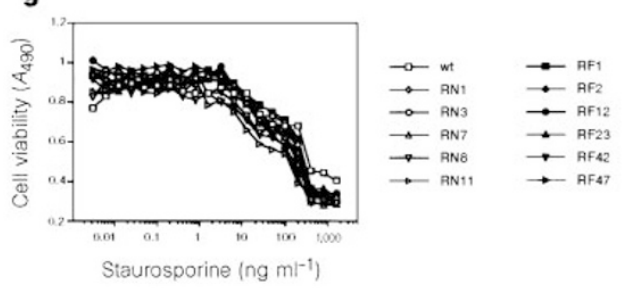

Figure 3 FLIPs protect eukaryotic cells from death receptor-induced apoptosis. a b. $293 T$ cells were transiently transfected with the indicated amounts of expression vectors for human Fas (a) or human TRAMP (b), together with human FLIP, FLIPS or FLIP and mock vector to keep the total amount of transfected DNA constant. Where indicated, $25 \mu \mathrm{M} z$-VAD-fmk was added to the cell culture after transfection. Quantitative analysis of cell death (induced by the overexpressed death receptors Fas or TRAMP in the absence of ligand) was done using a histone-DNA complex-release assay (the data shown are means \pm s.d., $n=3$ ). In a typical transfection experiment, $60-90 \%$ of cells underwent apoptosis upon transfection with death-receptor expression vectors. c, 293T cells were transiently transfected with the indicated amounts of an expression vector for the cytoplasmic portion of TNF-R1 together with FLIP $L$ and analysed as in a. d, Expression of FLIP $L$ and FLIPS in 293T cells. At high concentrations, FLIP $L$ causes cell death in the absence of death receptors. e, $\mathbf{f}$ Cellular extracts of VSV-FLIPL-transfected (FL), VSV-FLIPs-transfected (FS) and untransfected Jurkat (J) T-cell clones, Raji (R) B-cell clones and 293T cells were
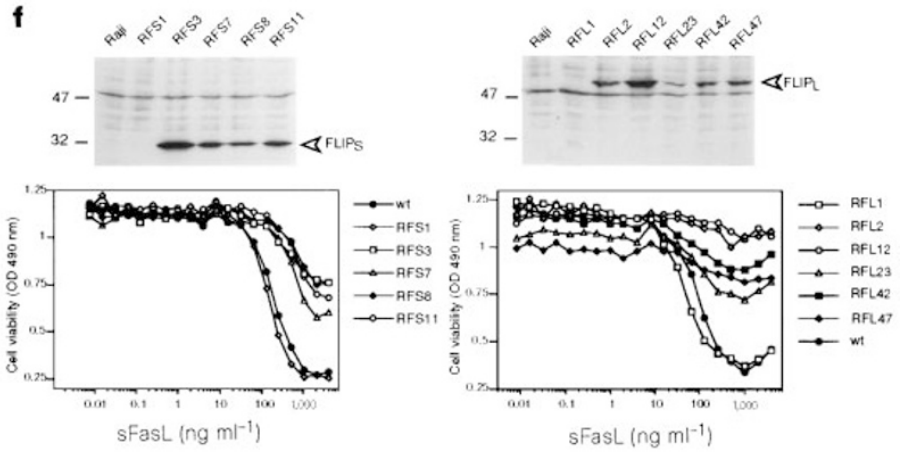

analysed for FLIP expression by anti-VSV western blotting (top). Migration positions of FLIPS and FLIP $L$ are indicated by open arrowheads. The top right panel of e shows a comparison of VSV-FLIPL expression in transiently transfected 293T cells (in the absence of Z-VAD-fmk) and two stably transfected lymphocyte clones. The arrow points to a protein detected nonspecifically, demonstrating equal protein loading. The susceptibility of the FLIP-transfected Jurkat (e) and Raji (f) clones to FasL-induced apoptosis was determined by incubating the cells for $20 \mathrm{~h}$ at $37^{\circ} \mathrm{C}$ with recombinant SFasL and subsequent determination of cell viability using a cell proliferation assay. e, FLIPL and FLIPS expressing Jurkat clones were incubated with the indicated concentrations of recombinant STRAIL for $20 \mathrm{~h}$ and cell viability was determined using a cell proliferation assay. At high concentrations of sFasL and STRAIL, $>95 \%$ of the untransfected cells underwent apoptosis. g. Staurosporine-induced cell death is not inhibited by FLIPs. Clones were treated with the indicated concentrations of staurosporine for $16 \mathrm{~h}$, and cell viability assayed using a cell proliferation assay. 

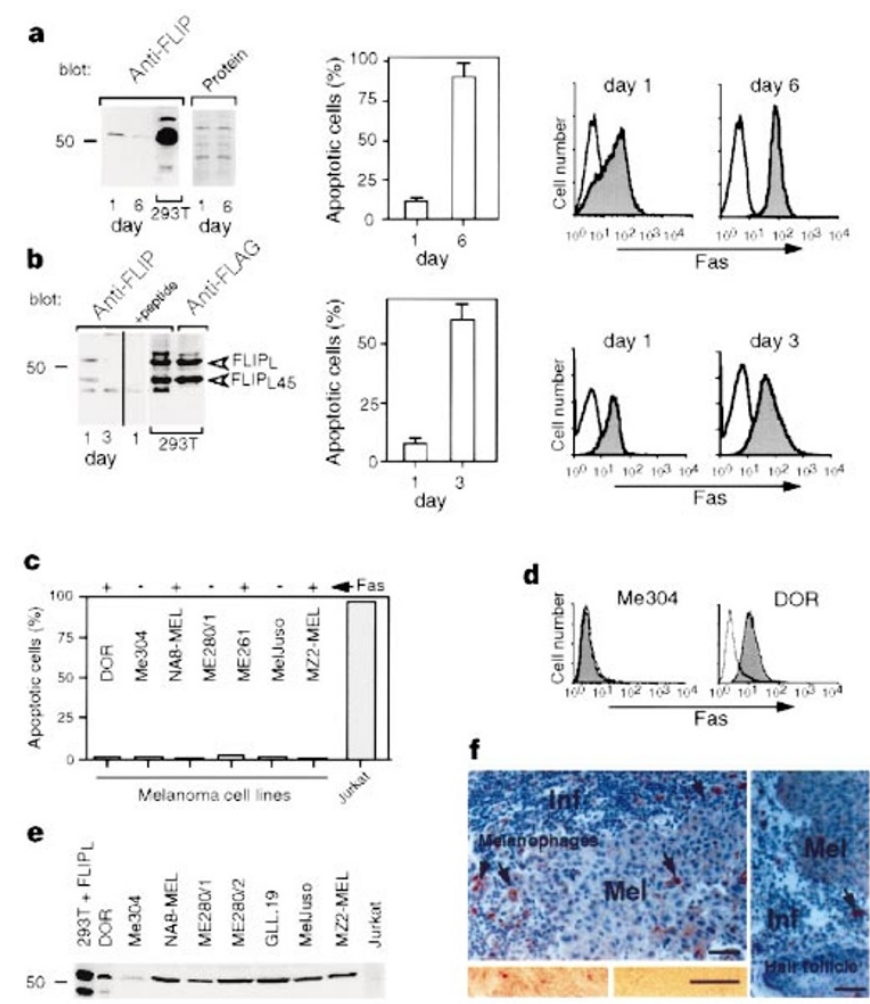

Figure 4 FLIP $P_{L}$ is expressed during T-cell activation and in human malignant melanoma. a, Western blot analysis of phytohaemaglutinin (PHA)-activated human T cells at day 1 and 6 of stimulation using the anti-FLIPAL109 antibody. A cell extract of FLIPL-transfected (0.3 $\mu \mathrm{g}$ DNA) 293T cells (in the presence of Z-VAD$\mathrm{fmk}$ ) is included as a control, and equal protein loading was verified by Ponceau $S$ staining of the nitrocellulose blot. The middle panel shows the susceptibility of day 1 and 6 activated T cells to apoptosis induced by treatment with sFasL $\left(0.2 \mu \mathrm{g} \mathrm{ml}^{-1}\right)$ for $6 \mathrm{~h}$. The percentage of apoptotic cells was determined by the histone-DNA complex-release assay. All data are given as mean \pm s.d. $(n=3)$. FACS analysis of Fas surface expression is shown on the right. Cells were stained with anti-Fas followed by an anti-mouse FITC-conjugated secondary antibody (filled curves) and analysed on a FACScan flow cytometer. Open curves represent cell staining with FITC-conjugated secondary antibody only. b. Western blot analysis of concanavalin A-activated mouse splenocytes at day 1 and 3 of stimulation. The quality of the anti-human FLIP antibody towards mouse FLIP was tested by comparing anti-FLIP and anti-Flag western blots of a cell extract from 293T cells transfected with a mouse Flag-FLIP expression vector in the absence of z-VAD-fmk. Moreover, in the western blot analysis of T cells from day 1 , $10 \mu \mathrm{g} \mathrm{ml}^{-1}$ of the peptide used for immunization was included as a specificity control. A band migrating at $\sim 41 \mathrm{~K}$ is detected nonspecifically and demonstrates equal protein loading. The middle panel shows the susceptibility of day 1 and 3 activated T cells to FasL-mediated apoptosis. Fas surface expression (right) was determined as in $\mathbf{a}$, except that the biotinylated anti-mouse Fas antibody Jo2 in combination with streptavidin-FITC was used for the detection of the antigen. $\mathbf{c}$, Melanoma cell lines resist killing by sFasL. Various cell lines were incubated with recombinant sFasL $\left(1 \mu \mathrm{g} \mathrm{ml}^{-1}\right)$ for $6 \mathrm{~h}$. The presence(+) or absence(-) of surface Fas was verified by FACS analysis (see Fig. 4d). FasL-sensitive Jurkat cells were used as control target cells. d, Fas surface expression on two selected melanoma cell lines (Me304 and DOR). Cells were stained as in a. e, Expression of FLIPL in melanoma cell lines. Anti-FLIP western blot analysis of melanoma cell lines derived from several patients with malignant melanoma. Controls included 293T cells transfected with a FLIPL expression vector and Jurkat cells. f, Expression of FLIP (brown) in two examples of human malignant melanoma (Mel, top and right panels). The tumours are surrounded by an inflammatory infiltrate (Inf). The dark cells (arrows) correspond to melanophages (macrophages with phagocytosed melanoma-derived melanin). The bottom sections from a metastatic melanoma were not counterstained with haematoxylin. The specificity of the reaction was assayed by the preincubation of the antibody with the peptide $\left(10 \mu \mathrm{g} \mathrm{ml}^{-1}\right)$ used for immunization (bottom right). Scale bars, $20 \mu \mathrm{m}$ each.

242-454 in frame with an N-terminal VSV epitope into a vector derived from PCR-3 (Invitrogen). The expression vector for HA-tagged FLICE was a gift from J. P. Medema and M. E. Peter (Heidelberg, Germany). Recombinant soluble FasL was from Alexis (San Diego). Additional expression vectors, cell lines and reagents used in this study have been described previously ${ }^{3,20}$. Melanoma cell lines were derived from tumour samples obtained from melanoma patients by surgical excision ${ }^{27}$. Metastatic melanomas of patients were removed by surgery and snap-frozen in liquid nitrogen-cooled isopentane. Sections ( $10 \mu \mathrm{m}$ thick) were cut and analysed as previously described ${ }^{27}$. Screening of cDNA libraries and northern blot analysis. cDNA clones for human FLIP $_{S}$ and FLIP $_{L}$ were isolated from a cDNA library of activated PBLs (a gift of H. Eibel, Freiburg) using a ${ }^{32}$ P-labelled DNA fragment (random primed labelling kit, Boehringer) encoding amino acids 1-170 of human FLIP and generated from EST clone 309776. A cDNA clone for murine FLIP $_{\mathrm{L}}$ was isolated from a murine heart muscle cDNA library (Stratagene) using the same probe. Northern blot analysis was performed by using human multiple tissue northern blots I and II (Clontech \#7760-1 and \#7759-1) according to the manufacturer's instructions. Antisense RNA probes containing the nucleotides corresponding to amino acids 1-170 (probe FLIP-N), 229-480 (FLIP-C) of FLIP , and $\beta$-actin were used.

Cell transfection, co-immunoprecipitation and western blotting. Stable transfections of Jurkat and Raji cells, transient transfection of 293T cells, immunoprecipitations and western blot analysis were done as previously described $^{3}$. Jurkat and Raji clones or 293T cells were checked for expression of transfected proteins by anti-tag western blot analysis of postnuclear cell lysates with equivalent protein content. 
Preparation and stimulation of Tcells. Isolation of splenocytes and enriched human $\mathrm{T}$ cells and their stimulation with concanavalin $\mathrm{A}$ and PHA were done as described $^{2,30}$.

Apoptosis and cell proliferation assays. Reduced cell proliferation or apoptosis as a result of death receptor stimulation or overexpression was measured using the Celltiter $96 \mathrm{AQ}$ proliferation assay (Promega) and the cell death detection ELISA (Boehringer Mannheim), respectively, as previously described $^{3}$.

Protease activity assays. Flag fusion proteins of the caspase homology region of FLIP $_{\mathrm{L}}$ and FLICE were generated in bacteria using modified pQE16 (Qiagen) bacterial expression vectors. Protease activity was determined on fluorogenic substrates as described ${ }^{7}$.

Chromosomal localization. A sequence-tagged site ${ }^{11}$ (A008B37) corresponding to FLIP maps to human chromosome 2 in the interval between the markers D2S116-D2S307. On the cytogenetic map, this interval corresponds to 2q33.

Received 19 May; accepted 11 June 1997.

1. Nagata, S. Apoptosis by death factor. Cell 88, 355-365 (1997)

2. Klas, C., Debatin, K. M., Jonker, R. R. \& Krammer, P. H. Activation interferes with the APO-1 pathway in mature human T cells. Int. Immunol. 5, 625-630 (1993).

. Thome, M. et al. Viral FLICE-inhibitory proteins (FLIPs) prevent apoptosis induced by death receptors. Nature 386, 517-521 (1997)

4. Boldin, M. P. et al. A novel protein that interacts with the death domain of Fas/APO1 contains a sequence motif related to the death domain. J. Biol. Chem. 270, 7795-7798 (1995).

5. Chinnaiyan, A. M., O'Rourke, K., Tewari, M. \& Dixit, V. M. FADD, a novel death domain-containing protein, interacts with the death domain of Fas and initiates apoptosis. Cell 81, 505-512 (1995).

6. Muzio, M. et al. FLICE, a novel FADD-homologous ICE/CED-3-like protease, is recruited to the CD95 (Fas/APO-1) death-inducing signaling complex. Cell 85, 817-827 (1996)

. Boldin, M. P., Goncharov, T. M., Goltsev, Y. V. \& Wallach, D. Involvement of MACH, a novel MORT1/ FADD-interacting protease, in Fas/APO-1- and TNF receptor-induced cell death. Cell 85, 803-815 (1996).

8. Bertin, J. et al. Death effector domain-containing herpesvirus and poxvirus proteins inhibit both Fasand TNFR1-induced apoptosis. Proc. Natl Acad. Sci. USA 94, 1172-1176 (1997).

9. Bucher, P., Karplus, K., Moeri, N. \& Hofmann, K. A flexible search technique based on generalized profiles. Comput. Chem. 20, 3-24 (1996).

10. Fernandes-Alnemri, T. et al. In vitro activation of CPP32 and Mch3 by Mch4, a novel human apoptotic cysteine protease containing two FADD-like domains. Proc. Natl Acad. Sci. USA 93, 7464 7469 (1996).

11. Schuler, G. D. et al. A gene map of the human genome. Science 274, 540-546 (1996).

12. Walker, N. P. C. et al. Crystal structure of the cysteine protease interleukin-1- $\beta$-converting enzyme-a (p20/p10)(2) homodimer. Cell 78, 343-352 (1994).

13. Wilson, K. P. et al. Structure and mechanism of interleukin- $1 \beta$ converting enzyme. Nature 370, 270 275 (1994)

14. Vincenz, C. \& Dixit, V. M. Fas-associated death domain protein interleukin-1 $\beta$-converting enzyme 2 (FLICE2), an ICE/Ced-3 homologue, is proximally involved in CD95- and p55-mediated death signaling. J. Biol. Chem. 272, 6578-6583 (1997).

15. Kischkel, F. C. et al. Cytotoxicity-dependent Apo-1 (Fas/CD95)-associated proteins form a deathinducing signaling complex (DISC) with the receptor. EMBO J. 14, 5579-5588 (1995).

16. Gu, Y. et al. Interleukin-1 $\beta$ converting enzyme requires oligomerization for activity of processed forms in vivo. EMBO J. 14, 1923-1931 (1995)

17. Medema, J. P. et al. FLICE is activated by association with the CD95 death-inducing signaling complex (DISC). EMBO J. 16, 2794-2804 (1997).

18. Bump, N. J. et al. Inhibition of ICE family proteases by baculovirus antiapoptotic protein $\mathrm{p} 35$. Science 269, 1885-1888 (1995)

19. Xue, D. \& Horvitz, H. R. Inhibition of the Caenorhabditis elegans cell-death protease CED-3 by a CED3 cleavage site in baculovirus p35 protein. Nature 377, 248-251 (1995).

20. Bodmer, J. L. et al. TRAMP, a novel apoptosis-mediating receptor with sequence homology to tumor necrosis factor receptor 1 and Fas(Apo-1/CD95). Immunity 6, 79-88 (1997).

21. Marsters, S. A. et al. Apo-3, a new member of the tumor necrosis factor receptor family, contains a death domain and activates apoptosis and NF-кB. Curr. Biol. 6, 1669-1676 (1996).

22. Kitson, J. et al. A death-domain-containing receptor that mediates apoptosis. Nature 384, 372-375 (1996).

23. Yu, G. L. et al. Signal transduction by DR3, a death domain-containing receptor related to TNFR-1 and CD95. Science 274, 990-992 (1996).

24. Tartaglia, L. A., Rothe, M., Hu, Y. F. \& Goeddel, D. V. Tumor necrosis factor's cytotoxic activity is signaled by the p55 TNF receptor. Cell 73, 213-216 (1993).

25. Pan, G. et al. The receptor for the cytotoxic ligand TRAIL. Science 276, 111-113 (1997).

26. Peter, M. E. et al. Resistance of cultured peripheral T cells towards activation-induced cell death involves a lack of recruitment of FLICE (MACH/caspase 8) to the CD95 death-inducing signaling complex. Eur. J. Immunol. 27, 1207-1212 (1997).

27. Hahne, M. et al. Melanoma cells express Fas(Apo-1/CD95) ligand: Implications for tumor immune escape. Science 267, 1363-1366 (1996)

28. Nagata, S. \& Golstein, P. The Fas death factor. Science 267, 1449-1456 (1995).

29. Francis, M. J. et al. Immunological evaluation of the multiple antigen peptide (MAP) system using the major immunogenic site of foot-and-mouth disease virus. Immunology 73, 249-254 (1991).

30. Lowin, B., Hahne, M., Mattmann, C. \& Tschopp, J. Cytolytic T-cell cytoxocity is mediated through perforin and Fas lytic pathways. Nature 370, 650-652 (1994).

Acknowledgements. We thank S. Belli for reading this manuscript; C. Servi for peptide synthesis; and R. Bullani, S. Hertig, M. Rousseaux and T. Bornand for technical assistance. This work was supported by grants of the Swiss National Science Foundation (J.T.) and the European Molecular Biology Organisation (to M.T.).

Correspondence and requests for material should be addressed to J.T. (e-mail: jurg.tschopp@ib.unil.ch) The Genbank accession numbers for FLIP cDNA sequences are U97074 (human FLIP L $_{\mathrm{L}}$ ), U97075 (human FLIP $_{\mathrm{S}}$ ), and U97076 (mouse $\operatorname{FLIP}_{\mathrm{L}}$ ).

\section{Tom5 functionally links mitochondrial preprotein receptors to the general import pore}

\section{Klaus Dietmeier*, Angelika Hönlinger*, Ulf Bömer, Peter J. T. Dekker, Christoph Eckerskorn $\dagger$, Fritz Lottspeich $†$, Michael Kübrich \& Nikolaus Pfanner}

Institut für Biochemie und Molekularbiologie, Universität Freiburg, Hermann-Herder-Strasse 7, D-79104 Freiburg, Germany

$\dagger$ Max-Planck-Institut für Biochemie, D-82152 Martinsried, Germany

* These authors contributed equally to this study.

Most mitochondrial proteins are synthesized as preproteins on cytosolic polysomes and are subsequently imported into the organelle ${ }^{1-3}$. The mitochondrial outer membrane contains a multisubunit preprotein translocase (Tom) which has receptors on the cytosolic side and a general import pore (GIP) in the membrane. Tom20-Tom 22 and Tom70-Tom37 function as import receptors ${ }^{4-7}$ with a preference for preproteins that have amino-terminal presequences or internal targeting information, respectively. Tom40 is an essential constituent of the $\mathrm{GIP}^{8,9}$, whereas Tom 6 and Tom 7 modulate the assembly and dissociation of the Tom machinery ${ }^{10,11}$. Here we report the identification of Tom5, a small subunit that has a crucial role importing preproteins destined for all four mitochondrial subcompartments. Tom 5 has a single membrane anchor and a cytosolic segment with a negative net charge, and accepts preproteins from the receptors and mediates their insertion into the GIP. We conclude that Tom5 represents a functional link between surface receptors and GIP, and is part of an 'acid chain's that guides the stepwise transport of positively charged mitochondrial targeting sequences.

For the preparation of Tom machinery, digitonin-lysed mitochondria from the yeast Saccharomyces cerevisiae were subjected to a coprecipitation with antibodies directed against Tom40. A noncharacterized band (labelled 'Tom5' in Fig. 1a, lane 2) was subjected to $\mathrm{N}$-terminal sequencing (Fig. $1 \mathrm{~b}$, sequence II). The sequence matched to a previously unknown open reading frame (ORF) on chromosome XVI (cosmid 9659, nucleotides 6,512-6,661), encoding a 50-residue protein of relative molecular mass $\left(M_{\mathrm{r}}\right) 5.98 \mathrm{~K}$ (Fig. 1b) which we term Tom5. Its sequence does not reveal significant homology to any known protein. The C-terminal half of Tom5 contains a hydrophobic segment (residues 27-45) of sufficient length to function as a membrane anchor. This segment is flanked by positively charged residues that may interact with negatively charged headgroups of membrane phospholipids. The $\mathrm{N}$-terminal portion of Tom 5 carries a negative net charge (Fig. 1b).

Pure outer-membrane vesicles ${ }^{12,13}$ contained a prominent band with an electrophoretic mobility like that of Tom5 (Fig. 1a, lane 1), and N-terminal amino-acid sequencing confirmed that it was indeed Tom5 (Fig. 1b, sequence I). An antiserum directed against an N-terminal peptide of Tom5 was generated (Fig. 1a, lane 3). To assess whether Tom 5 is an integral membrane protein, mitochondria were treated with sodium carbonate at $\mathrm{pH} 11.5$ (ref. 11). This treatment allows the extraction of soluble and peripheral membrane proteins (Fig. 1c, columns 3 and 5) but leaves integral membrane proteins in the membrane sheets (Fig. 1c, columns 2 and 4). Tom5 was fully resistant to extraction by sodium carbonate (Fig. 1c, column 1), indicating that it is an integral membrane protein. Tom 5 was also resistant to treatment of mitochondria with trypsin at all concentrations tested (Fig. 1d, lanes 2-6), even after lysis of the mitochondria with Triton X-100 (Fig. 1d, lane 7). Treatment of 